\title{
AVALIAÇÃO MORFOFUNCIONAL DO AUTO-IMPLANTE OVARIANO NO RETROPERITÔNIO
}

\author{
MORPHOFUNCTION ASSESSMENT OF OVARIAN AUTOTRANSPLANTATION IN THE RETROPERITONEUM
}

\author{
Luiz R. Alberti'; Leonardo S. Vasconcellos ${ }^{1}$; Andy Petroianu² \& Maurício B. Nunes³
}

\begin{abstract}
${ }^{1}$ Acadêmicos de Medicina da Faculdade de Ciências Médicas de Minas Gerais. ${ }^{2}$ Docente Titular do Departamento de Cirurgia da Faculdade de Medicina - Universidade Federal de Minas Gerais, Docente Livre da Faculdade de Medicina de Ribeirão Preto - USP, Docente Livre da Escola Paulista de Medicina - UNIFESP, Doutor em Fisiologia e Farmacologia, Pesquisador IA do CNPq. ${ }^{3}$ Anatomopatologista do Instituto Moacyr Junqueira e da Santa Casa de Belo Horizonte.

CorRespondência: Prof. Andy Petroianu - Av. Afonso Pena, n 1626, Apto 1901. CEP 30130-005, Belo Horizonte, Minas Gerais. E-mail: petroian@medicina.ufmg.br - Fone (fax) (31) 3274-7744
\end{abstract}

ALBERTI LR; VASCONCELLOS LS; PETROIANU A \& NUNES MB. Avaliação morfofuncional do auto-implante ovariano no retroperitônio Medicina, Ribeirão Preto, 35: 30-35, jan./mar. 2002.

RESUMO: Introdução: Em muitos procedimentos pélvicos e abdominais, a ooforectomia bilateral se impõe, porém suas complicações sistêmicas, como insuficiências hormonais, podem ser de difícil controle.

Objetivo: Visando a preservação da função gonadal, em casos de ooforectomia, avaliaram-se aspectos funcionais e histológicos de tecido ovariano auto-implantado, em posição heterotópica.

Material e Métodos: Foram selecionadas 36 ratas Wistar, com ciclos estrais, normais, divididas aleatoriamente em quatro grupos $(n=9)$ : $G 1$ - controle- submetido a laparotomia, sem procedimento cirúrgico adicional; G2- ooforectomia bilateral; G3- os ovários retirados foram implantados integralmente no retroperitônio; G4- os ovários retirados foram fatiados e também implantados no retroperitônio. Nos $3^{\circ}$ e $6^{\circ}$ meses pós-operatórios, realizaram-se esfregaços vaginais e estudos histológicos dos implantes ovarianos.

Resultados: Os animais do grupo G1 tiveram seus ciclos normalmente. As ratas dos grupos G2 permaneceram durante todo o período em diestro. No grupo G3, no sexto mês, duas ratas tiveram ciclos completos, compatíveis com a fase estral; três animais apresentaram ciclos irregulares e os restantes permaneceram em diestro. No grupo G4, no sexto mês pós-operatório, três ratas apresentaram ciclos incompletos, cinco ratas apresentaram ciclos estrais completos, e apenas uma permaneceu fixa em diestro. O estudo anatomopatológico confirmou viabilidade ovariana em ambos os grupos de auto-implante (G3 e G4) com melhores resultados para o G4.

Conclusões: O auto-implante ovariano no retroperitônio, na forma fatiada, apresentou meIhor preservação morfofuncional do que a do íntegro.

UNITERMOS: Ovário. Transplante. Esfregaço Vaginal. Ovariectomia. Rata.

\section{INTRODUÇÃO}

A irradiação pélvica e a quimioterapia, notadamente na doença de Hodgkin, além de outras neoplasias malignas, tais como as leucemias na infância, resultam em castração em mais de $80 \%$ das pacientes tratadas, mesmo quando se realiza ooforopexia ${ }^{(1)}$. Em muitos casos, realiza-se ooforectomia por necessidade, em procedimentos sobre a pelve ${ }^{(2)}$.

O ovário é responsável por mais de $50 \%$ da produção de testosterona na pós-menopausa. Um declínio súbito de andrógenos, em decorrência da ooforectomia, pode levar à diminuição da libido e provocar osteoporose, além de alterações nos níveis de lipoproteínas e 
aumento do risco de doenças cardiovasculares ${ }^{(3,4,5)}$. Além disso, estudos mostram baixos índices de aderência à terapia hormonal por longos períodos ${ }^{(6)}$.

Uma alternativa mais fisiológica de manter a função hormonal em mulheres que precisam de se submeter à retirada de ovários normais e ainda funcionantes, é o transplante ovariano heterotópi$\mathrm{co}^{(7,8,9)}$. Diversos trabalhos vêm procurando descobrir o melhor local para realizar o auto-implante ovariano ${ }^{(3,8,10}$, $)$, bem como a forma mais adequada de tal procedimento para preservar a função ovariana ${ }^{(3,11)}$.

O presente estudo propõe a realização de autoimplante ovariano no retroperitônio, por considerar essa região a mais adequada sob aspecto de drenagem venosa para a veia cava inferior, que recebe fisiologicamente o sangue e os hormônios dos ovários através das veias ovarianas. A capacidade de secreção hormonal dos ovários foi avaliada pela manutenção do ciclo estral, com base em esfregaços vaginais. A viabilidade e as alterações morfológicas foram estudadas por método histológico.

\section{MATERIAL E MÉTODOS}

Este trabalho experimental foi realizado no período de agosto de 2000 a fevereiro de 2001, de acordo com as recomendações das Declarações de Helsinque e as Normas Internacionais de Proteção aos Animais $^{(12,13)}$, e foi aprovado por Comissão de Ética da Universidade Federal de Minas Gerais.

O presente estudo foi conduzido em 36 ratas Wistar adultas, pesando entre 250 e 280 gramas, com aproximadamente 120 dias de idade. Para verificar se os animais apresentavam ciclos estrais regulares, esfregaços vaginais foram colhidos diariamente, durante um período de sete dias. As ratas com ciclos estrais atípicos foram excluídas.

Os procedimentos foram realizados sob anestesia com éter sulfúrico. As ratas foram divididas aleatoriamente em quatro grupos $(\mathrm{n}=9)$ :

Grupo 1 (G1) - controle - laparotomia e identificação dos ovários;

Grupo 2 (G2) - ooforectomia bilateral;

Grupo 3 (G3) - ooforectomia bilateral e transplante dos ovários íntegros para o retroperitônio, logo abaixo dos rins, utilizando-se fio de prolene 5-0;

Grupo 4 (G4) - ooforectomia bilateral e transplante dos ovários seccionados em três fatias transversais, de aproximadamente 3 milímetros de espessura, para o retroperitônio, logo abaixo dos rins, utilizando-se fio de prolene 5-0.
$\mathrm{O}$ útero e as trompas permaneceram in situ, em todos os grupos. Após as cirurgias, os animais receberam água e ração para ratos ad libitum. Eles foram observados diariamente, com vista a sinais de anormalidades locais ou sistêmicas, durante 180 dias.

Uma semana antes e durante todo o período posterior ao ato cirúrgico, os animais foram mantidos em ambiente com controle de luminosidade de 12 horas/dia.

No terceiro e no sexto mês pós-operatórios, avaliou-se a função ovariana por meio de esfregaços vaginais diários, com base no ciclo estral, durante um período de 10 dias. $\mathrm{O}$ animais foram imobilizados e, usando-se uma pipeta, introduziu-se, na cavidade vaginal da rata, $0,25 \mathrm{ml}$ de solução salina a $0,5 \%$. Após lavar a cavidade vaginal duas vezes com solução salina a $0,9 \%$, o fluido foi retirado com o auxílio de outra pipeta limpa. Caso houvesse contaminação, repetia-se o lavado. Cada ponteira foi usada uma única vez para cada exame.

Os esfregaços foram avaliados a fresco, sem coloração, e conduzidos sob aumento de 40X e 100X, para observação das características celulares, de acordo com o ciclo ovariano, com base em estudos iniciais publicados por Cooper et al. ${ }^{(14)}$, em cobaias, e por Long \& Evans ${ }^{(15)}$, em ratas. Esses autores observaram que a relação entre o estrogênio ovariano e a citologia vaginal, em ratas, é representada por um ciclo ovariano com duração média de quatro dias, dividido em três fases distintas $(14,15,16)$ :

diestro - caracteriza-se por concentrações baixas de estrogênio e esfregaços vaginais com predomínio de leucócitos e algumas células epiteliais corneificadas em permeio; esta fase é considerada como préovulatória;

proestro - há um aumento da concentração do estradiol, e, no esfregaço vaginal, predominam células polimorfonucleadas, dispersadas ou acumuladas; esta é a fase em que ocorre a ovulação;

estro - há queda dos níveis de estradiol e predomínio de células epiteliais, escamosas ,corneificadas; esta é a fase pós-ovulatória.

Após a determinação dos ciclos estrais, no sexto mês pós-operatório, os animais foram mortos com dose excessiva de éter sulfúrico. Os ovários implantados foram removidos e preparados para exame histológico de rotina, utilizando-se a coloração pela hematoxilina e eosina. Os parâmetros histológicos avaliados para comparação entre as duas formas de implante ovariano, íntegra e fatiada, foram: degeneração, fibrose, reação inflamatória, angiogênese, cistos foliculares, desenvolvimento folicular e corpos lúteos. 
Os resultados foram comparados por meio do teste $t$ de Student e quiquadrado. As diferenças foram consideradas significativas para valores correspondentes a $\mathrm{P}<0,05$.

\section{RESULTADOS}

Todos os animais sobreviveram até o sexto mês pós-operatório, sem complicações cirúrgicas. A Tabela I apresenta os achados citológicos vaginais de todos os grupos em ambos os períodos avaliados, $3^{\circ} \mathrm{e}$ $6^{\mathrm{o}}$ meses pós-operatórios.

No G1 (controle), as ratas tiveram a seqüência do ciclo regular (diestro, proestro, estro) tanto no terceiro quanto no sexto mês pós-operatório.

No G2 (ooforectomia bilateral), todos os animais permaneceram em diestro durante os 14 dias do estudo, em ambos os períodos de análise. As ratas deste grupo foram consideradas como não produtoras de hormônio ovariano.

No G3 (transplante íntegro), duas ratas tiveram ciclos completos compatíveis com a fase estral, no sexto mês avaliado. Três animais apresentaram ciclos irregulares e as restantes permaneceram em diestro. A função ovariana foi considerada inadequada nos animais deste grupo, exceto nas duas ratas que apresentaram ciclos estrais completos e regulares.

No G4 (transplante fatiado), três ratas apresentaram ciclos incompletos, mostrando fases compatíveis com estro e diestro, no sexto mês pós-operatório, sendo consideradas como produtoras deficientes de hormônios sexuais. Cinco ratas apresentaram ciclos estrais completos, sendo consideradas como portado- ras de ovários auto-implantados funcionantes, e apenas uma permaneceu fixa em diestro.

$\mathrm{Na}$ segunda laparotomia, a cavidade abdominal mostrava-se de aspecto normal. Os ovários implantados estavam preservados, sem sinais de reação inflamatória local ou reações aparentes ao fio cirúrgico.

Os estudos anatomopatológicos, realizados no sexto mês pós-operatório, revelaram viabilidade ovariana nos três grupos com ovário. Os ovários do grupo-controle foram todos normais. Já no Grupo 3 (Figura 1a), identificou-se infiltrado inflamatório, moderado, composto de células mononucleares e fibrose discreta, além de neoformação vascular. Observaramse áreas de isquemia e necrose de leve intensidade nos ovários pertencentes às ratas que estavam fixas em diestro. Nos outros animais, não houve sinais de isquemia ou necrose. Corpos lúteos e cistos foliculares semelhantes aos dos ovários normais foram encontrados em proporções variáveis.

No Grupo 4 (Figura 1b), também se observou viabilidade ovariana, com fibrose e inflamação de graus indo de leve a moderado. Áreas de isquemia ocorreram apenas na parte periférica do ovário da rata que não saiu da fase de diestro. Cistos foliculares e corpos lúteos também foram identificados. Os cistos foliculares foram maiores do que os encontrados nos grupos 1 e 3 .

As ratas submetidas a implantes heterotópicos de ovário na forma fatiada (grupo G4) apresentaram melhor padrão de ciclo estral e aspecto histológico mais próximo ao normal do que as dos ovários íntegros (Grupo G3) $(\mathrm{p}<0,05)$.

\begin{tabular}{|c|c|c|c|c|c|c|c|c|}
\hline \multirow{2}{*}{ RATAS } & \multicolumn{2}{|c|}{ GRUPO 1} & \multicolumn{2}{|c|}{ GRUPO 2} & \multicolumn{2}{|c|}{ GRUPO 3 * } & \multicolumn{2}{|c|}{ GRUPO 4 * } \\
\hline & $3^{\circ}$ mês & $6^{\circ}$ mês & $3^{\circ}$ mês & $6^{\circ}$ mês & $3^{\circ}$ mês & $6^{\circ}$ mês & $3^{\circ}$ mês & $6^{\circ}$ mês \\
\hline 1 & $\mathrm{C}$ & $\mathrm{C}$ & A & A & A & A & C & $\mathrm{C}$ \\
\hline 2 & $\mathrm{C}$ & C & A & A & 1 & 1 & $\mathrm{C}$ & C \\
\hline 3 & $\mathrm{C}$ & $\mathrm{C}$ & A & A & $\mathrm{C}$ & $\mathrm{C}$ & A & 1 \\
\hline 4 & $\mathrm{C}$ & C & A & A & A & A & $\mathrm{C}$ & C \\
\hline 5 & $\mathrm{C}$ & $\mathrm{C}$ & A & A & 1 & 1 & A & I \\
\hline 6 & C & C & A & A & 1 & 1 & C & C \\
\hline 7 & C & C & A & A & A & A & A & I \\
\hline 8 & C & C & A & A & C & C & A & A \\
\hline 9 & C & C & A & A & A & A & C & C \\
\hline \multicolumn{9}{|c|}{$\begin{array}{l}\text { Leqenda: Grupo } 1 \text { - controle. Grupo } 2 \text { - ooforectomizadas. Grupo } 3 \text { - transplante de ovário ínteqro para o retroperitônio. Grupo } 4 \text { - } \\
\text { transplante de ovário na fom a fatiada para o retroperitônio. C - Cido completo. A - Cido ausente. I - Cido incompleto. } \\
\text { * Diferentes entre si para p<0,05 (teste quiquadrado) }\end{array}$} \\
\hline
\end{tabular}




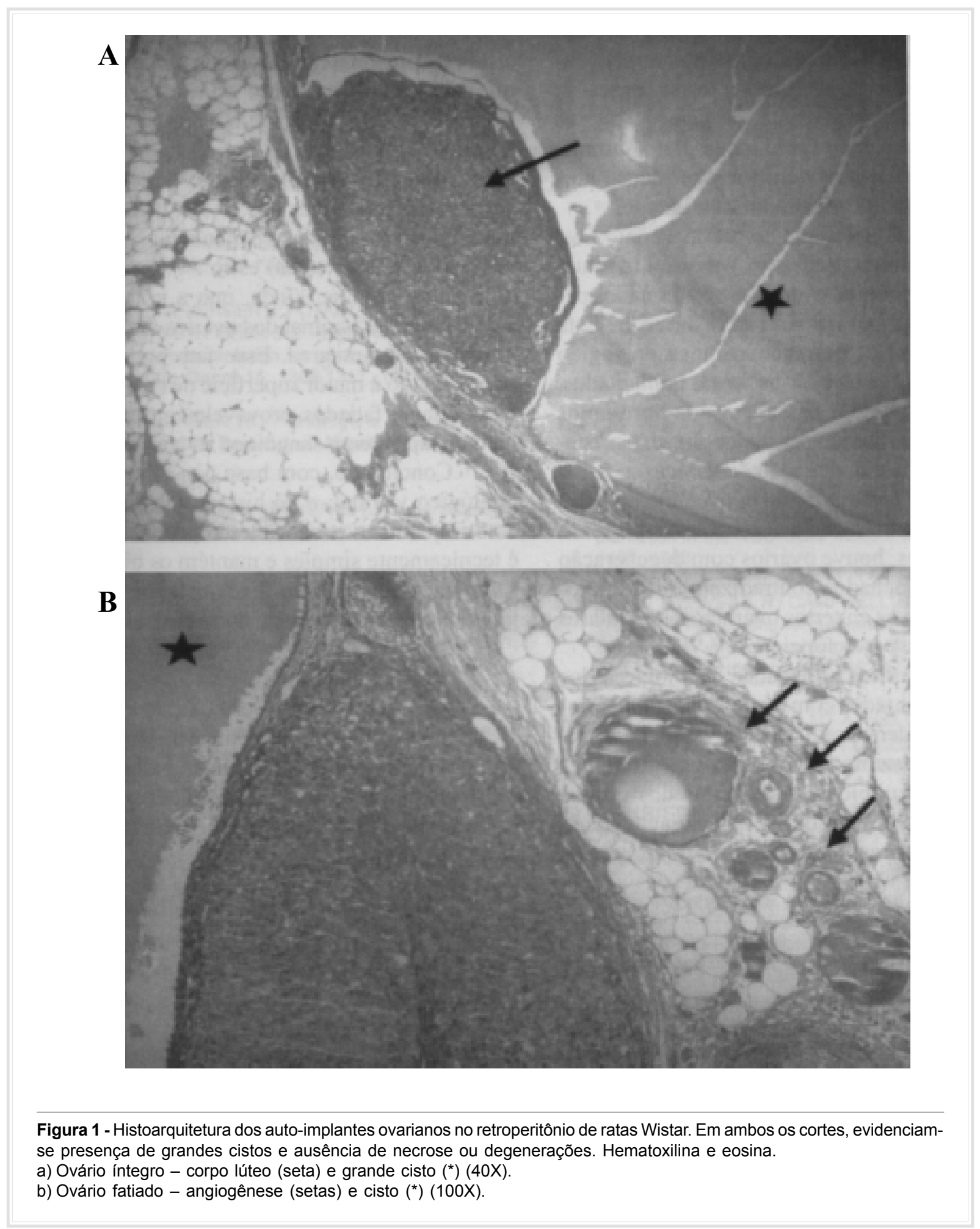

\section{DISCUSSÃO}

O transplante ovariano visa à preservação de sua função e à diminuição dos efeitos indesejáveis do climatério precoce. Apesar dos múltiplos estudos, ainda não se definiu o melhor local para a implantação dos ovários $^{(1,2,4,7,17)}$.

A avaliação do local de implantação dos ovários baseou-se na viabilidade da histoarquitetura e função ovariana. Verificou-se indiretamente a produção 
hormonal com base na citologia obtida pelos esfregaços vaginais ${ }^{(14,15,16)}$. Os auto-implantes ovarianos foram considerados funcionais, quando as ratas apresentaram ciclo estral normal, ou seja, apresentando seqüência típica de todas as fases: diestro, estro, proestro. Nas ratas com ciclo permanente, na fase em diestro ou que apresentaram ciclo estral incompleto, durante os dez dias avaliados, considerou-se a produção hormonal dos ovários auto-implantados deficiente.

A implantação ovariana no retroperitônio teve como base o padrão da drenagem venosa. Em situação normal, os hormônios ovarianos são drenados pelas veias ovarianas para a veia cava inferior. Esta mesma veia recebe a drenagem venosa da região retroperitoneal em que os ovários foram implantados. Dessa forma, evitou-se a passagem dos hormônios pelo fígado, onde poderiam ser metabolizados precocemente e interferir em sua ação sistêmica.

No presente trabalho, não houve adversidades decorrentes da vascularização dos enxertos, pois, em nenhum dos grupos, houve ovários com degeneração histológica. Esses achados contradizem outros autores que consideram os enxertos ovarianos avasculares inadequados sob aspecto funcional ${ }^{(18,19,20)}$.

Ao relacionarmos os achados histológicos dos ovários auto-implantados com a citologia vaginal, todos os animais evidenciaram aspecto vaginal compatível com função hormonal ovariana em diferentes fases. Os aspectos citológicos das ratas dos grupos em que se realizou implante de tecido ovariano fatiado (G4) apresentaram resultados melhores do que os obtidos quando se implantava o ovário íntegro.

Tomou-se o cuidado de manter controlada a luminosidade ambiente, porque ciclos ovarianos consistentes podem ser detectados em roedores apenas quando abrigados sobre condições regulares de luminosidade. Fotoperíodos padronizados têm sido definidos como sendo de 12 ou 14 horas de exposição à luz comum. Alterações desses períodos podem levar a várias alterações dos ciclos, incluindo até mesmo inibição da ovulação e manutenção de esfregaços vaginais persistentes em estro ${ }^{(14,18)}$.

Observou-se, ainda, que a eficácia funcional sobre a mucosa vaginal dos ovários fatiados foi maior do que a dos íntegros. Esse fato pode ter sido, em parte, devido à maior superfície de contato dos enxertos ovarianos fatiados, provavelmente proporcionando melhor suprimento sangüíneo ao tecido ovariano.

Concluindo, com base nos aspectos cirúrgico, citológico vaginal, e de histoarquitetura ovariana, o auto-implante ovariano heterotópico no retroperitônio é tecnicamente simples e mantém os ovários viáveis. Entretanto, sob aspecto funcional, apenas os ovários fatiados tiveram atividade hormonal adequada.

\section{AGRADECIMENTOS}

Somos gratos ao acadêmico de Medicina, Juliano Ferreira Barbosa, por auxílio no trabalho em laboratório. Agradecemos também ao CNPq e FAPEMIG pelos auxílios financeiros que permitiram a realização deste trabalho.

ALBERTI LR; VASCONCELLOS LS; PETROIANU A \& NUNES MB. Morphofunction assessment of ovarian autotransplantation in the retroperitoneum. Medicina, Ribeirão Preto, 35: 30-35, jan./march 2002.

ABSTRACT: Introduction: Oophorectomy is required in many pelvic and abdominal procedures. The endocrine disturbances may be followed by severe systemic complications.

Objective: In order to maintain the ovarian function after oophorectomy, the present study assessed ovarian autotransplantation in the retroperitoneum.

Material and Methods: Thirty-six cycling female Wistar rats were randomly divided into four groups: G1 (control) - sham operation; G2 - bilateral oophorectomy; G3 - bilateral oophorectomy and integre ovarium autotransplantation in the retroperitoneum; G4 - bilateral oophorectomy followed by sliced ovarium autotransplantation in the retroperitoneum. The estrous cycle was investigated in the third and sixth months. Hystologic studies of the ovaries were carried out.

Results: The animals of group G1 preserved the cycling sequence. The rats of group G2 remained in diestrus. In group G3, the vaginal smear of two rats showed pattern of estrus, three showed irregular cycles, and the other animals remained in diestrus. In group G4, the rats showed irregular cycles, five presented normal cycle sequence and only one rat remained in diestrous. The histology indicated ovarian viability in Groups 3 and 4, and better aspect in Group 4.

Conclusions: Sliced ovarian autotransplantation in the retroperitoneum showed better morphofunction preservation.

UNITERMS: Ovary. Transplantation. Vaginal Smears. Ovariectomy. Rat. 


\section{REFERÊNCIAS BIBLIOGRÁFICAS}

1 - THEOBALD PV; ROFFÉ JL; BERROCAL J; PORRIER ML; LÉVY $G$ \& MULLER $G$. Autotransplantation ovarienne hétérotopique chez la femme. Presse Med 16: 1239-1242, 1987.

2 - CORLETA HVE; CORLETA O; CAPP E \& EDELWEISS MI. Subcutaneous autologous ovarian transplantation in Wistar rats maintains hormone secretion. Fertil Steril 70: 16-19, 1998.

3 - SPEROFF T; DWASON NV; SPEROFF L \& HABER RJ. A riskbenefit analysis of elective bilateral oophorectomy. Am J Obstet Gynecol 164: 165-174, 1991.

4 - STIFF PJ; BAYER R; KERGER C; POTKUL RK; MALHOTRAD; PEACE DJ; SMITHD \& FISHER SG. High-dose chemotherapy with autologous transplantation for persistent/relapsed ovarian cancer. J Clin Oncol 15: 1309-1317, 1997.

5 - DAVIS SR \& BUERGER HG. Androgens and postmenopausal woman. J Clin Endocrinol Metab 81: 2759-2763, 1996.

6 - ERAKER SA; KIRSCHT JP \& BECKER MH. Understsanding and improving patient compliance. Ann Intern Med 100: 258-268, 1984.

7 - VON THEOBALD P; ROFFÉ JL; BERROCAL J; LE PORRIER M; LÉVY G \& MULLER G. Autotransplantation ovarienne hétérotopique chez la femme. Presse Med 16: 1239-1241, 1987.

8 - CANDY CJ; WOOD MJ \& WHITTINGHAM DG. Follicular development in cryopreserved marmoset ovarian tissue after transplantation. Hum Reprod 10: 2334-2338, 1995.

9 - QUATTROPANI SL. Ovulation of ovarian implants in unilaterally ovariectomized rats. Anat Record 209:331-336, 1984.

10 - GUANASENA KT; VILLINES PM; CRISTER ES \& CRITSER JK. Live births after autologous transplant of cryopreserved mouse ovaries. Hum Reprod 12: 101-106, 1997.
11 - DZIUK PJ; PARVIZI N \& ELLENDORFF F. Concentrations of steroids in jugular and hepatic portal veins of pigs after ingestion of testosterone, estrogen, or progesterone or transplantation of ovaries to the intestine. Domest Anim Endocrinol 17: 29-38, 1999.

12 - COOPER JE. Ethics and laboratory animals. Vet Rec 116: 594-595, 1985.

13 - Petroianu A. Pesquisa experimental. In: Petroianu A, ed. Ética, moral e deontologia médicas, Guanabara Koogan, Rio de Janeiro, p.185-190, 2000.

14 -COOPER RI; GOLDMAN JM \& VANDENBERGH JG. Monitoring of the estrus cycle in the laboratory rodent by vaginal lavage. Fem Reprod Toxicol 3: 45-56, 1993.

15 - LONG JA \& EVANS HM. The estrous cycle in rat and its associated phenomena. Am J Physiol, 32: 936-942, 1959.

16 - GAY VL \& TOMACARI RL. Follicle-stimulating hormone secretion in the female rat. Science 184: 75-76, 1974.

17 - WEISSMAN A; GOTLIEB L \& COLGAN T. Preliminarry experience with subcutaneous human ovarian cortex transplantation in the NOD-SCID mouse. Biol Reprod 60: 14621467, 1999.

18 - BAIRD DT; WEBB R; CAMPBELL BK; HARKNESS LM \& GOSDEN RG. Long-term ovarian function in sheep after ovariectomy and transplantation of autografts stored at 196 C. Endocrinology 140: 462-471, 1999.

19 - CALLEJO J; JAUREGUI MT \& VALLS C. Heterotopic ovarian transplantation without vascular pedicle in syngeneic Lewis rats. Fertil Steril 72: 513-527, 1999.

20-HARRISON FA; CHAMBERS SG \& GREEN EA. Autotransplantation of the ovary to the neck in the sow. J Endocrinol 83: 46-47, 1980.

Recebido para publicação em 18/07/2001

Aprovado para publicação em 12/12/2001 